\title{
GROUPS WITH A CYCLIC SYLOW SUBGROUP
}

\author{
WALTER FEIT ${ }^{1)}$ \\ Dedicated to the memory of Tadasi Nakayama
}

\section{$\S 1$. Introduction}

By focussing attention on indecomposable modular representations J. G. Thompson [11] has recently simplified and generalized some classical results of R. Brauer [1] concerning groups which have a Sylow group of prime order. In this paper this approach will be used to prove some results which generalize theorems of R. Brauer [2] and H. F. Tuan [12].

We will say that a finite group $(B)$ is of type $L_{2}(p)$ if every composition factor is either a $p$-group or a $p^{\prime}$-group or is isomorphic to $P S L_{2}(p)$. Thus in particular every $p$-solvable group is of type $L_{2}(p)$. It is well known that every subgroup of a group of type $L_{2}(p)$ is again of type $L_{2}(p)$.

Theorem 1. Let $\mathbb{B}$ be a finite group with a cyclic $S_{p}$-subgroup $\mathfrak{P}$ for some prime p. Assume that $\mathbb{B S}$ is not of type $L_{2}(p)$. Suppose that $\mathbb{B}$ has a faithful indecomposable representation $\mathfrak{\&}$ of degree $d \leq p$ in a field of characteristic $p$. Then $p \neq 2, \quad|\mathfrak{B}|=p, \quad \mathfrak{Q} \mid \mathfrak{P} \quad$ is indecomposable and $\mathbf{C}_{\mathfrak{S}}(\mathfrak{P})=\mathfrak{P} \times \mathbf{Z}(\mathfrak{S})$. Furthermore $d \geq 2 / 3(p-1)$ and $d \geq \frac{7}{10} p-\frac{1}{2}$ in case $p \geq 13$.

It is known [9] that the multiplier of $\mathfrak{H}_{5}, \mathfrak{H}_{6}, \mathfrak{P}_{7}$, respectively has a nontrivial complex representation of degree 2, 3, 4 respectively. Hence this is the case in any algebraically closed field. The new simple group discovered by $Z$. Janko [8] has a 7-dimensional representation in the field of 11 elements. Thus for $p \leq 11$ the estimate in Theorem 1 is the best possible (since $d$ is an integer). However it follows easily from the last statement that $d \geq 2 / 3(p-1)$ is never the best possible estimate for $p \geq 13$. By modifying the argument in section 4 slightly it can be shown that for $p \geq 13$ the estimate can be improved

Received July 2, 1965.

1) The work on this paper was partially supported by the U. S. Army Contract DA31-124-ARO-D-336. 
provided $\left|\mathbf{N}_{\mathscr{S}}(\mathfrak{B}): \mathbf{C}_{\mathscr{S}}(\mathfrak{P})\right|$ is sufficiently large. In particular it is easy to show that if $\mathbb{B}=\mathbb{S}^{\prime},\left|\mathbf{N}_{\mathscr{S}}(\mathfrak{B}): \mathbf{C}_{\mathscr{S}}(\mathfrak{B})\right|=p-1$ and $p \geq 13$ then $d \geq \frac{3(p-1)}{4}$. This is in sharp contrast to the case of Janko's group where $p=11, d=7$ and $\left|\mathbf{N}_{\mathfrak{S}}(\mathfrak{B}): \mathbf{C}_{\mathfrak{S}}(\mathfrak{B})\right|=10$. It would be of interest to determine the best possible lower bound for $d$ in case $p \geq 13$. Since the Symmetric group on $p$ letters has a faithful representation of degree $p-2$ in the field of $p$ elements one cannot do better than $p-3$. However this is probably much too large in general.

Theorem 1 is easily seen to imply some results of Brauer [2] and Tuan [12] concerning groups 8 which have a faithful irreducible complex representations of "small" degree relative to the size of some prime dividing $|\mathbb{\$}|$. As another application of these methods the following can be proved.

Theorem 2. Suppose the $S_{p}$-subgroup $\mathfrak{P}$ of $\mathbb{B}$ is not normal in $\mathbb{B}$ and $\mathbf{Z}(\mathbb{B})$ $=\langle 1\rangle$. Assume that $\mathbb{B}$ has a complex irreducible representation of degree $d$ with $\frac{p-1}{2}<d<p-1$. Let $\left|\mathbf{N}_{\mathfrak{S}}(\mathfrak{B}): \mathbf{C}_{\mathfrak{S}}(\mathfrak{B})\right|=e$. Then $\mathbb{S}$ is simple and $e \equiv \frac{p-1}{e} \equiv 0$ $(\bmod 2)$. Thus in particular $p \equiv 1(\bmod 4)$.

The only known groups which satisfy the hypotheses of Theorem 2 are $P S L_{:}(p)$ with $\left.p \equiv 1 \cdot \bmod 4\right)$ and $d-1=e=\frac{p-1}{2}$, and $P S L_{2}(p-1)$ where $p-1$ $=2^{a}$ for some integer $a>1$ with $e=2$ and $d=p-2$.

\section{$\S$ 2. Preliminaries}

Let $K$ be a field and $\mathbb{S}$ a group. If $M, N$ are $K \$ 3$-modules then $M+N$ denotes their direct sum and $a M=M+\cdots+M a$ times for any nonnegative integer $a$. The kernel of $M$ is the kernel of the representation of $\&$ corresponding to $M$. If $\mathscr{S}$ is a subgroup of $\mathbb{S}$ then $M \mid \mathfrak{F}$ denotes the restriction of

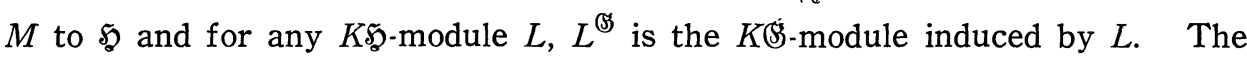
contragradient module of $M$ is denoted by $M^{*}$. The remainder of the notation and terminology is standard.

Basic properties of modules will be used continually. In particular the Mackey decomposition [3, (44.2)] and a fundamental result of D. G. Higman $[3,(63.5)]$ are of importance. Also a theorem of Schanuel will be used [6, (1.6 e) $]$ or $[10$, p. 270]. The following result is a simple consequence of the Mackey decomposition, the proof of [3, (51.2)] and Fitting's Lemma.

(2.1) Suppose that $K$ is a field of characteristic $p$. Let $\mathfrak{P}$ be a p-group and $\mathfrak{\$}$ 


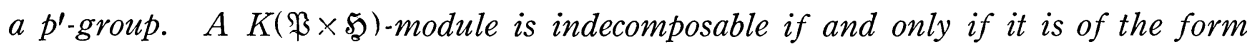

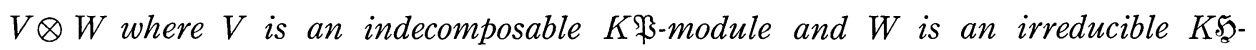
module.

An exposition of the fundamentals of the theory of blocks can be found in $[3$, Chapter XII]. The following special cases of some results of R. Brauer [2] will be needed.

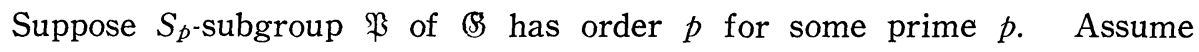
further that $\mathbf{C}_{\mathfrak{S}}(\mathfrak{B})=\mathfrak{B} \times \mathbf{Z}(\mathfrak{B})$. Let $e=\left|\mathbf{N}_{\mathfrak{S}}(\mathfrak{B}): \mathbf{C}_{\mathscr{S}}(\mathfrak{P})\right|$.

(2.2) If $\zeta$ is an irreducible complex character of \& with $1<\zeta(1)<p-1$ then $e<p-1$ and either $\zeta(1)=e$ or $\zeta(1)=p-e$. In the latter case $\zeta$ does not contain the principal Brauer character as a modular constituent. Furthermore if $B$ is the $p$-block of $\$$ containing $\zeta$ then $B$ contains exactly $\frac{p-1}{e}$ irreducible complex character of degree $5(1)$, any two of which are p-conjugate and hence coincide as Brauer characters.

(2.3) If $\mathbf{Z}(\mathbb{B})=\langle 1\rangle$ and $e=2$ then the degree of any irreducible modular representation of $(\mathbb{S}$ is $1, p-2$ or at least $p$.

The following result of Tuan [12, Theorem C] is also useful.

(2.4) Any modular irreducible representation of $\$$ in the principal block can be written in the field of $p$ elements.

The proofs of (2.2), (2.3) and (2.4) can be simplified considerably using the methods of [11].

\section{§. Local Results}

Throughout this section $K$ is a field of characteristic $p$. $\quad F \Re$ is a Frobenius group with Frobenius kernel $\mathfrak{P}$ where $|\mathfrak{P}|=p$ and $\mathfrak{F} \cap \mathfrak{P}=\langle 1\rangle$. The one dimensional $K$-representation $\alpha$ of $\mathbb{E P}$ is defined by

$$
G^{-1} P G=P^{\alpha(G)} \text { for } P \in \mathfrak{P}, G \in \mathfrak{F} \text {. }
$$

The following result is a reformulation of [11, Lemma 2].

Lemma 3.1. Let $\lambda$ be a one dimensional $K$-representation of $\mathfrak{P}$ and let $1 \leq$ $s \leq p$. Then there exists an indecomposable Kßङ-module $V_{s}^{\lambda}$ such that $\operatorname{dim}_{K} V_{s}^{\lambda}=$ $s, V_{s \mid{ }_{B}}^{\lambda}$ is indecomposable and if $U$ is the unique submodule of $V_{s}^{\lambda}$ with $\operatorname{dim}_{K} U$ 
$=1$ then $u G=\lambda(G) u$ for all $u \in U, G \in[$. F. Furthermore every nonzero indecomposable K\$(5-module is isomorphic to some $V_{s}^{\lambda} ; V_{s}^{\lambda} \approx V_{t}^{\mu}$ if and only if $s=t$, $\lambda=\mu ; V_{s}^{\lambda}$ is projective if and only if $s=p$.

Throughout this section $V_{s}^{\lambda}$ will be defined as in Lemma 3.1 and for any $\lambda, V_{0}^{\lambda}=0$. In case $\left[E=\langle 1\rangle\right.$ we will write $V_{s}=V_{s}^{\lambda}$. If $E \in \mathbb{E}$ then $\operatorname{det}_{s}^{\lambda}(E)$ denotes the determinant of $E$ acting as a linear transformation on $V_{s}^{\lambda}$ and $\varphi_{s}^{\lambda}$ denotes the Brauer character of $\mathfrak{P} \in$ corresponding to $V_{s}^{\lambda}$.

Lemma 3.2. Let $0 \leq i \leq s \leq p$. Then $V_{s}^{\lambda}$ has a unique submodule $U_{i}$ with $\operatorname{dim}_{K} U_{i}$ $=i$. Furthermore $U_{i} \approx V_{i}^{\lambda}$ and $V_{s}^{\lambda} / U_{i} \approx V_{s-i}^{\lambda \alpha}$.

Proof. Since every irreducible $K \mathfrak{P}$ (5-module is 1-dimensional $V_{s}^{\lambda}$ has an $i$ dimensional submodule $U_{i}$ for $0 \leq i \leq s$. As $V_{s \mid \Re}^{\lambda}$ is indecomposable each $U_{i}$ is uniquely determined. By Lemma 3.1. $U_{1} \subseteq U_{i}$ and so $U_{i} \approx V_{i}^{\lambda}$.

If $i=0$ or $i=s$ the last statement is clear. Suppose that $i=1$ and $s \geq 2$. Since $\mid\left[||(p-1)\right.$ the $K$ \&-module $U_{2} \mid \mathfrak{E}$ is a direct sum of two $K$ - -modules. Choose a $K$-basis $x, y$ of $U_{2}$ such that $y \in U_{1}$ and $x E=\mu(E) x$ for all $E \in($ and some 1-dimensional $K$-representation of $\mathfrak{F}$. Then for suitable $P \in \mathfrak{P}, x P=x+y$. Thus for $E \in \mathbb{E}$

$$
\begin{aligned}
x+\alpha(E) y & =x P^{\alpha(E)}=x E^{-1} P E=\mu\left(E^{-1}\right) x P E=\mu\left(E^{-1}\right) x E+\mu\left(E^{-1}\right) y E \\
& =x+\mu\left(E^{-1}\right) \lambda(E) y .
\end{aligned}
$$

Hence $\mu(E)=\lambda(E) \alpha^{-1}(E)$ for all $E \in\left[\right.$. If $\bar{x}$ denotes the image of $x$ in $V_{s}^{\lambda} / U_{1}$ this implies that if $G=P E, P \in \mathfrak{B}, E \in(E$ then

$$
\bar{x} G=\bar{x} E=\lambda \alpha^{-1}(E) \bar{x}=\lambda \alpha^{-1}(G) \bar{x}
$$

Thus $V_{s}^{\lambda} / U_{i} \approx V_{s-1}^{\lambda \alpha^{-1}}$. Since $V_{s}^{\lambda} / U_{1} \approx\left(V_{s}^{\lambda} / U_{1}\right) /\left(U_{i} / U_{1}\right)$ for $i \geq 1$ the result follows by induction on $i$.

Lemma 3.3. $\left(V_{s}^{\lambda}\right)^{*} \approx V_{s}^{\lambda^{-1} \alpha^{(s-1)}} . \quad \operatorname{det}_{s}^{\lambda}(E)=\lambda^{s} \alpha^{-s(s-1) / 2}(E)$ for $E \in(\xi . \quad$ Let $\xi$ $=\left\langle E_{0}\right\rangle$. Then $\varphi_{s}^{\alpha j}\left(E_{0}\right)=\varepsilon^{j}\left(\sum_{i=0}^{s-1} \varepsilon^{-i}\right)$ for a suitable primitive $|\xi|$ th root of unity $\varepsilon$ and all $s$ and $j$.

Proof. This is an immediate consequence of Lemma 3.2.

Leмma 3.4. $V_{s}^{\lambda} \otimes V_{p}^{\mu} \approx \sum_{i=0}^{s-1} V_{p}^{\lambda \mu \alpha-i}$ for $0 \leq s \leq p$. 
Proof. Let $M_{\mu}$ be the 1-dimensional $K \&$-module corresponding to the representation $\mu \mid \xi$. It is easily seen (and well known) that $V_{p}^{\mu} \approx M_{\mu}^{\mathfrak{F}}$. By Lemma $3.2 V_{s \mid}^{\lambda} \mid \& \approx \sum_{i=0}^{s-1} M_{\lambda \alpha^{-i}}$. Thus [3, p. 325].

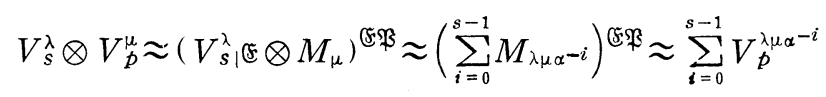

Lemma 3.5. If $0 \leq s \leq t$ and $s+t \leq p$ then

$$
V_{s}^{\lambda} \otimes V_{t}^{\mu} \approx \sum_{i=0}^{s-1} V_{s+t-1-2 i}^{\lambda \mu \alpha-i}
$$

Proof. It suffices to prove the result in case $\mid[|\xi|=p-1$. If $s=0$ or 1 it is immediate.

Suppose $s=2$. By [6, Theorem $3(2.3 \mathrm{~b})] V_{2} \otimes V_{t} \approx V_{t-1}+V_{t+1}$. Thus by Lemma $3.1 V_{2}^{\lambda} \otimes V_{t}^{\mu} \approx V_{t-1}^{3}+V_{t+1}^{\gamma}$ for some $\beta, \gamma$. By Lemma 3.2 there exist $K$-bases $\left\{x_{0}, x_{1}\right\}$ of $V_{2}^{\lambda}$ and $\left\{y_{0}, \ldots, y_{t-1}\right\}$ of $V_{t}^{\mu}$ such that for $E \in E$ and all $i$

$$
x_{i} E=\lambda \alpha^{-i}(E) x_{i}, y_{i} E=\mu \alpha^{-i}(E) y_{i} .
$$

Furthermore if $U$ is the submodule of $V_{2}^{\lambda} \otimes V_{t}^{\mu}$ consisting of all $u$ with $u P=u$ for all $P \in \mathfrak{P}$ then $\operatorname{dim}_{K} U=2$. Let $P \in \mathfrak{P}$. Then there exist $a, b \in K$ with $a b$ $\neq 0$ such that

$$
\begin{array}{ll}
x_{0} P=x_{0}, & x_{1} P=x_{1}+a x_{0} \\
y_{0} P=y_{0}, & y_{1} P=y_{1}+b y_{0}
\end{array}
$$

Define

$$
v_{0}=x_{0} \otimes y_{0}, \quad v_{1}=\frac{1}{a} x_{1} \otimes y_{0}-\frac{1}{b} x_{0} \otimes y_{1} .
$$

Then $v_{i} P=v_{i}$ for $i=0,1$, and so $\left\{v_{0}, v_{1}\right\}$ is a basis of $U$. If $E \in \mathbb{E}$ then

$$
v_{0} E=\lambda \mu(E) v_{0}, \quad v_{1} E=\lambda \mu \alpha^{-1}(E) v_{1}
$$

As $|\mathfrak{E}| \neq 1, \lambda \mu \neq \lambda \mu \alpha^{-1}$. Therefore $v_{0} \in V_{t-1}^{\beta}$ and $\beta=\lambda \mu$ or $v_{0} \in V_{t+1}^{\gamma}$ and $\gamma=\lambda \mu$.

Let $\left\langle x_{0}\right\rangle \approx V_{1}^{\lambda}$ be the submodule of $V_{2}^{\lambda}$ generated by $x_{0}$. Let $W=\left\langle x_{0}\right\rangle \otimes V_{t}^{u}$ $\approx V_{t}^{\mu \lambda}$. Thus $W$ is indecomposable and $v_{0} \in W$. Since $\operatorname{dim}_{K} W=t$ it follows that $W \cap U_{t+1} \neq 0$, where $U_{t+1}$ is a submodule of $V_{2}^{\lambda} \otimes V_{t}^{\mu}$ with $U_{t+1} \approx V_{t+1}^{\gamma}$. By Lemma $3.2 v_{0} \in W \cap U_{t+1}$. Hence $v_{0} \in U \cap U_{t+1}$ and $\gamma=\lambda \mu$. Thus $\beta=\lambda \mu \alpha^{-1}$ and the result is proved in case $s=2$.

We proceed by induction on $s$. Assume that $s \geq 3$ and the result has been proved for $s-1$ and $s-2$. Then 


$$
V_{s-1}^{\lambda} \otimes V_{2}^{1} \otimes V_{t}^{\mu} \approx\left(V_{s-2}^{\lambda \alpha^{-1}} \otimes V_{t}^{\mu}\right)+\left(V_{s}^{\lambda} \otimes V_{t}^{\mu}\right)
$$

Thus by induction

$$
\sum_{i=0}^{s-2}\left(V_{s+t-2-2 i}^{\lambda \mu \alpha^{-i}} \otimes V_{2}^{1}\right) \approx \sum_{i=0}^{s-3} V_{s+t-3-2 i}^{\lambda \mu \alpha^{-i-1}}+\left(V_{s}^{\lambda} \otimes V_{t}^{\mu}\right)
$$

Applying the first part of the lemma once again yields that

$$
V_{s+t-1}^{\lambda \mu}+2\left(\sum_{i=1}^{s-2} V_{s+t-1-2 i}^{\lambda \mu \alpha-i}\right)+V_{t-s+1}^{\lambda \mu \alpha-(s-1)} \approx \sum_{i=0}^{s-3} V_{s+t-3-2 i}^{\lambda \mu \alpha-i-1}+\left(V_{s}^{\lambda} \otimes V_{t}^{\mu}\right) .
$$

The result now follows from the Krull-Schmidt Theorem.

The next result is proved in a similar manner to $[6,(2.5 \mathrm{a})]$.

Lemma 3.6. Suppose that $1 \leq b, c \leq p-1$ and $V_{b}^{\beta} \otimes V_{c}^{\gamma} \approx \sum_{i=0}^{k} V_{e_{i}}^{\sigma_{i}}$ with $e_{i}>0$ for $i=0, \ldots, k$. Then

$$
\sum_{i=0}^{k} V_{p}^{q_{i} \alpha^{b-e_{i}}}+\left(V_{p-b}^{\beta} \otimes V_{c}^{\Upsilon}\right) \approx \sum_{j=0}^{c-1} V_{p}^{\beta \gamma \alpha-j}+\sum_{i=0}^{k} V_{p-e_{i}}^{g_{i j} b-e_{i}} .
$$

Proof. By Lemma 3.2

$$
0 \rightarrow V_{p-b}^{\beta \alpha p-b} \rightarrow V_{p}^{\beta \alpha p-b} \rightarrow V_{b}^{\beta} \rightarrow 0 .
$$

is exact. Tensoring with $V_{c}^{\Upsilon}$ yields that

$$
0 \rightarrow V_{p-b}^{\beta \alpha} p-b \otimes V_{c}^{\Upsilon} \rightarrow V_{p}^{\beta \alpha p-b} \otimes V_{c}^{\Upsilon} \rightarrow V_{b}^{\beta} \otimes V_{c}^{\Upsilon} \rightarrow 0
$$

is exact. Also

$$
0 \rightarrow \sum_{i=0}^{k} V_{p-e_{i}}^{\tau_{i \alpha}^{p-e_{i}}} \rightarrow \sum_{i=0}^{k} V_{p}^{\beta_{i} \alpha^{p-e_{i}}} \rightarrow \sum_{i=0}^{k} V_{e_{i}}^{\beta_{i}} \rightarrow 0
$$

is exact. Thus Schanuel's Theorem and Lemma 3.4 imply that

$$
\sum_{i=0}^{k} V_{p}^{\tau_{i}^{\alpha} \alpha^{p-e_{i}}}+\left(V_{p-b}^{\beta \alpha p-b} \otimes V_{c}^{\Upsilon}\right) \approx \sum_{j=0}^{c-1} V_{p}^{\beta \gamma \alpha p-l-\jmath}+\sum_{i=0}^{k} V_{p-e_{i}}^{\gamma_{i \alpha} p-e_{i}}
$$

The result follows by tensoring this equation with $V_{1}^{\alpha^{b-p}}$

Lемма 3.7. If $1 \leq s \leq \frac{p-1}{2}$ then

$$
\begin{gathered}
V_{s}^{\lambda} \otimes V_{s}^{\mu} \approx \sum_{i=0}^{s-1} V_{2 i+1}^{\lambda \mu \alpha^{i+1-s}} \\
V_{p-s}^{\lambda} \otimes V_{p-s}^{\mu} \approx \sum_{i=0}^{s-1} V_{2 i+1}^{\lambda \mu \alpha^{s+i}}+\sum_{i=2 s}^{p-1} V_{p}^{\lambda \mu \alpha^{i}}
\end{gathered}
$$


Proof. The first statement is a special case of Lemma 3.5. Also Lemma 3.5 yields that

$$
V_{s}^{\lambda} \otimes V_{p-s}^{\mu} \approx \sum_{i=0}^{s-1} V_{p-1-2 i}^{\lambda \mu \alpha-i}
$$

Apply Lemma 3.6 with $\beta=\lambda, \gamma=\mu, b=s$ and $c=p-s$. Then

$$
\sum_{i=0}^{s-1} V_{p}^{\lambda \mu \alpha^{s+i-p+1}}+\left(V_{p-s}^{\lambda} \otimes V_{p-s}^{\mu}\right) \approx \sum_{j=0}^{p-s-1} V_{p}^{\lambda \mu \alpha-j}+\sum_{i=0}^{s-1} V_{2 i+1}^{\lambda \mu \alpha^{s+i-p+1}}
$$

Since $\alpha^{p-1}(G)=1$ for all $G \in \mathfrak{F} \mathfrak{P}$ the Krull Schmidt Theorem implies the result.

Lemma 3.8. If $1 \leq s \leq \frac{p-1}{2}$ then

$$
\begin{gathered}
V_{s}^{\lambda} \otimes\left(V_{s}^{\lambda}\right)^{*} \approx \sum_{i=0}^{s-1} V_{2 i+1}^{\alpha^{i}} \\
V_{p-s}^{\lambda} \otimes\left(V_{p-s}^{\lambda}\right)^{*} \approx \sum_{i=0}^{s-1} V_{2 i+1}^{\alpha^{i}}+\sum_{i=s}^{p-s-1} V_{p}^{\alpha^{i}}
\end{gathered}
$$

Proof. This follows directly from Lemmas 3.3 and 3.7 and the fact that $\alpha^{p-1}(G)=1$ for all $G \in \mathfrak{F}$.

\section{$\S$ 4. Proof of Theorem 1}

Throughout this section $\&$ is a group which satisfies the hypotheses of Theorem $1 . \mathfrak{F}$ is a $S_{p}$-subgroup of $\mathbb{S}$. Since $d \leq p$ in Theorem $1 \mathfrak{P}$ has exponent $p$ and so $|\mathfrak{B}|=p$ as $\mathfrak{B}$ is cyclic. $\mathfrak{R}=\mathbf{N}_{\mathscr{S}}(\mathfrak{B})$ and $\mathfrak{S}=\mathbf{C}_{\mathfrak{G}}(\mathfrak{B})=\mathfrak{B} \times \mathfrak{F}$. By assumption $\mathfrak{R} \neq \mathbb{S}$ and by Burnside's transfer theorem $\mathfrak{R} \neq \mathbb{E}$. K is a field of characteristic $p$.

$\mathscr{M}=\left\{M \mid M\right.$ is an indecomposable $K \$$-module with $\operatorname{dim}_{K} M \leq p$ and $\mathfrak{P}$ is not in the kernel of $M\}$.

By assumption $\mathscr{M}$ is nonempty. If $M \in \mathscr{M}$ then $M$ is a direct summand of $\left(M_{\mid \mathfrak{R}}\right)^{\stackrel{\Im}{5}}$ by D. G. Higman's Theorem [3. $\left.\S 63\right]$. Thus $M_{\mid \mathfrak{R}}$ is indecomposable by the Mackey decomposition and if $\operatorname{dim}_{\kappa} M<p$ then $M$ is uniquely determined

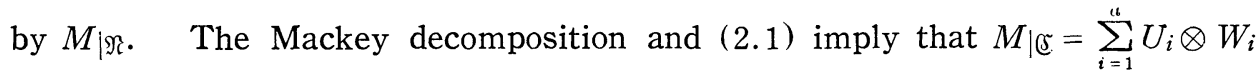
where for each $i U_{i}$ is an indecomposable $K \mathfrak{P}$-module and $W_{i}$ is an irreducible

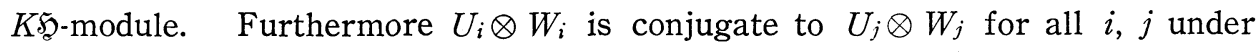
the action of $\mathfrak{R} / \mathcal{C}$. Thus $\operatorname{dim}_{K} U_{i}=c, \operatorname{dim}_{K} W_{i}=b$ are both independent of $i$ and in the notation of section $3 U_{i} \approx V_{c}$ for all $i$. Therefore 


$$
M_{\mid \mathfrak{c}} \approx V_{c} \otimes\left(\sum_{i=1}^{a} W_{i}\right), \operatorname{dim}_{K} W_{i}=b .
$$

The triple $a=a(M), b=b(M), c=c(M)$ is a set of invariants attached to $M$ and (4.1) implies that

$$
\operatorname{dim}_{K} M=a(M) b(M) c(M) .
$$

Lemma 4.1. Suppose that $p \geq 5$. If $M \in \mathscr{M}$ then $\operatorname{dim}_{K} M>2$.

Proof. Suppose $\operatorname{dim}_{K} M \leq 2$ for some $M \in \mathscr{M}$. Let $\AA$ be the kernel of $M$. Then $\mathbb{B} / \Re$ is isomorphic to a subgroup of $G L_{2}(K)$. All finite subgroups of $G L_{2}(K)$ are known and it is easily seen that $\mathbb{S} / \AA$ and hence $\mathbb{B}$, is of type $L_{2}(p)$ contrary to assumption.

Lemma 4.2. Suppose that $p \geq 5$. If $M \in \mathscr{M}$ with $\mathfrak{S}$ in the kernel of $M$ then $\operatorname{dim}_{K} M>3$.

Proof. Let $M \in \mathscr{M}$ with $\dot{\mathscr{S}}$ in the kernel of $M$. Suppose that $\operatorname{dim}_{K} M \leq 3$. By Lemma 4.1 it may be assumed that $\operatorname{dim}_{K} M=3$ and $M$ is absolutely irreducible. We will reach a contradiction by showing that $\mathbb{S}$ is of type $L_{2}(p)$. By changing notation it may be assumed that $\mathcal{B}^{\prime}=\mathbb{B}$ and $M$ is faithful. Thus $\mathbf{C}_{\mathfrak{S}}(\mathfrak{B})=\mathfrak{P}$. Let $\Re=\mathfrak{P} \mathbb{E}$ with $\mathfrak{P} \cap \mathbb{E}=\langle 1\rangle$. Let $\mathbb{E}=\langle E\rangle$. Let $\alpha$ be defined as

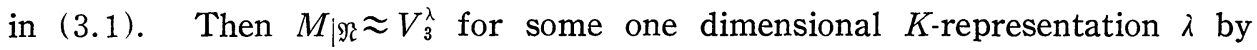
Lemma 3.1 and (4.1). Lemmas $3.1,3.3$ and 3.8 imply that $M \otimes M^{*}=L_{0}+$ $L_{1}+L_{2}$ where $\operatorname{dim}_{K} L_{i}=2 i+1$ and $L_{i}\left|\stackrel{*}{\mathfrak{k}}=L_{i}\right| \Re$. Thus $M$ may be chosen so that $M \mid * M_{\mid \Re}^{*} \approx$

Since $\mathbf{C}_{\mathscr{S}}(\mathfrak{B})=\mathfrak{P}$ there is only one block of defect $1[3,(86.10)]$. Hence $M$ is in the principal block of $\mathbb{B}$. Thus if $K_{0}$ is the field of $p$ elements there exists a $K_{0}$-representation $\tilde{F}$ of $\&$ corresponding to $M$ by (2.4). Since $M_{\mid \Re} \approx$ $\left.M\right|_{\mathfrak{F}} ^{*}$ it follows from Lemma 3.3 that $\tilde{F}$ is equivalent to $\tilde{F}^{*}$. An argument of R. Brauer [2, p. 438] now implies that $\&$ is isomorphic to a subgroup of $O_{3}(p)$. Since $O_{3}(p)$ is of type $L_{2}(p)$ so is $\&$ contrary to assumption.

Lemma 4.3. Suppose that $p \geq 5$. If $M \in \mathscr{M}$ then $c(M)>\frac{p-1}{2}$.

Proof. Suppose $M \in \mathscr{K}$ with $c=c(M) \leq \frac{p-1}{2}$. By Lemma 3.8 and (4.1)

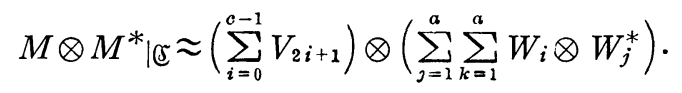


Thus no direct summand of $M \otimes M^{*} \mid \mathfrak{E}$ is projective. Let $W_{0}$ be the trivial 1 dimensional $K \$$-module. Then

$$
M \otimes M^{*} \mid \Subset \approx \sum_{i=0}^{c-1}\left(V_{2 i+1} \otimes W_{0}\right)+U
$$

where $U$ is a direct sum of indecomposable modules none of which are projective. Since $M \in \mathscr{M}, c>1$. Thus $V_{3} \otimes W_{0}$ is isomorphic to a direct summand of $M \otimes M^{*} \mid c$. Let $L$ be a direct summand of $M \otimes M^{*}$ such that $V_{3} \otimes W_{0}$ is isomorphic to a direct summand of $L_{j e}$. Since no direct summand of $L_{\mid \mathfrak{R}}$ is projective, $\left.L\right|_{\mathfrak{N}}$ is indecomposable. As $\mathscr{S}$ is in the kernel of $V_{3} \otimes W_{0} \mathscr{S}$ is also in the kernel of $L_{\mid \Re}$. Thus $L / \widetilde{c}$ is indecomposable by Lemma 3.1. Hence $\operatorname{dim}_{K} L=3$ contrary to Lemma 4.2 .

Lemma 4.4. If $M \in \mathscr{M},\left.M\right|_{\mathfrak{B}}$ is indecomposable and $\mathfrak{g}=\mathbf{Z}(\mathbb{S})$.

Proof. If $\operatorname{dim}_{K} M=p$ then $M$ is projective and so $M \mid \Re_{\beta}$ is projective and hence indecomposable. Suppose that $\operatorname{dim}_{K} M \leq p-1$. If $p=3$ then $M$ is indecomposable since $\mathfrak{P}$ is not in the kernel of $M$. If $p \geq 5$ then (4.2) and Lemma 4.3 imply that $a(M)=b(M)=1$. Thus by (4.1) $M_{\mid \Re}$ is indecomposable in any case. If $\tilde{F}$ is the $K$-representation of $\mathbb{S}$ corresponding to $M$ this implies that any $\not^{\prime}$-element in the commuting ring of $\tilde{\xi} \mid \mathfrak{B}$ is a scalar. Thus $\mathscr{H}=\mathbf{Z}(\mathbb{B})$ as required.

The proof of Theorem 1 can now be given. If $p=2$ then $\&$ is 2 -solvable since $|\mathfrak{S}|=2$ contrary to assumption. Thus $p \neq 2$. In view of Lemma 4.4 it only remains to prove the inequalities. If $p=3$ the result is trivial and if $p$ $=5$ it follows from Lemma 4.1. Hence it may be assumed that $p \geq 7$. It may further be assumed that $\mathbb{B}=\left(\mathcal{S}^{\prime}\right.$ and $K$ is algebraically closed without loss of generality.

Choose $L \in \mathscr{M}$ with $\operatorname{dim}_{K} L$ minimal. Let $d=p-s=\operatorname{dim}_{K} L$. It may be assumed that $L$ is faithful. By Lemma 4.3 and (4.1)

$$
L_{i c} \approx V_{p-s} \otimes W, \operatorname{dim}_{K} W=1, s \leq \frac{p-1}{2}
$$

Since $\mathfrak{N} / \mathfrak{S}$ is cyclic and $\mathscr{H} \subseteq \mathbf{Z}(\mathfrak{R})$ it follows that $\mathfrak{N} / \mathfrak{P}$ is abelian. Thus there exists a $K Y_{i-m o d u l e} W_{1}$ whose kernel contains $\mathfrak{P}$ such that $W_{1 \mid \mathfrak{H}}=W$. Then

$$
L_{\mid \mathfrak{R}} \otimes L_{\mid \mathfrak{R}}^{*} \approx\left(L_{\mathfrak{R}} \otimes W_{1}^{*}\right) \otimes\left(\left.L\right|_{\mathfrak{R}} \otimes W_{1}^{*}\right)^{*} .
$$


Furthermore

$$
\left(L_{\mid \mathfrak{R}} \otimes W_{1}^{*}\right)_{\mid \mathfrak{E}} \approx V_{p-s} \otimes W_{0}
$$

where $W_{0}$ denotes the trivial 1-dimensional $K$ $_{2}$-module. Let $\overline{\mathfrak{N}}=\mathfrak{N} / \mathfrak{S}$. Thus $L \mid \mathfrak{R} \otimes W_{1}^{*}$ is a $K \bar{\Re}$-module. Hence (4.3), (4.4) and Lemma $3.8 \mathrm{imply}$ that in the notation of section 3

$$
\left.L_{\mid \mathfrak{R}} \otimes L^{*}\right|_{\mathfrak{R}} \approx \sum_{i=0}^{s-1} V_{2 i+1}^{\alpha^{i}}+\sum_{i=s}^{p-s-1} V_{p}^{\alpha^{2}}
$$

where each $V_{j}^{\lambda}$ is a $K \overline{\mathfrak{\Re}}$-module.

Higman's Theorem and (4.5) imply that

$$
L \otimes L^{*} \approx \sum_{i=0}^{s-1} L_{i}+A
$$

where each $L_{i}$ is indecomposable, $A$ is projective and $L_{i \mid \Re}$ has $V_{2 i+1}^{\alpha^{i}}$ has a direct summand. Let

$$
L_{i \mid \mathfrak{R}}=V_{2 i+1}^{\alpha^{i}}+\sum_{j=1}^{m_{i}} V_{p}^{\mu_{i j}}
$$

Thus $L_{0}$ is the 1-dimensional trivial $K \$$-module. By (4.5)

$$
\left\{\mu_{i j} \mid j=1, \ldots, m_{i} ; i=0, \ldots, s-1\right\} \subseteq\left\{\alpha^{i} \mid s \leq i \leq p-s-1\right\}
$$

Suppose that $p-s<2 / 3(p-1)$. Then $p<3 s-1$. By (4.7)

$$
\sum_{i=1}^{s-1} m_{i} \leq(p-s-1)-s+1=p-2 s<s-1
$$

Hence at least $(s-1)-(p-2 s)$ of the $m_{i}$ are zero. Thus $m_{k}=0$ for some $k$ with

$$
1 \leq k \leq(s-1)-\{(s-1)-(p-2 s)\}=p-2 s .
$$

Thus by (4.6)

$$
\operatorname{dim}_{K} L_{k}=2 k+1 \leq 2 p-4 s+1=(p-s)+(p+1-3 s)<p-s=d .
$$

Hence $L_{k} \in \mathscr{M}$ contrary to the minimality of $d$. Therefore in proving Theorem 1 it may be assumed that $p \geq 13$ and $d=p-s \geq 2 / 3(p-1)$ or equivalently

$$
s \leq \frac{p+2}{3}, p \geq 13
$$


Choose $E \in \mathbb{B}$ so that $\mathfrak{N}=\langle E, \mathbb{F}\rangle$. Since $\mathbb{B}=\mathbb{B}^{\prime} E$ must have determinant 1 when considered as a linear transformation on the $K$-space $L_{i}$ for $i=0, \ldots, s-1$. Thus by (4.6) and Lemma 3.3.

$$
\left(\prod_{\jmath=1}^{m_{i}} \mu_{i j}\right) \alpha^{m_{i}(p-1) / 3}(E)=\left(\prod_{\jmath=1}^{m_{i}} \mu_{i j}\right) \alpha^{-m_{i} p(p-1) / 2}(E)=1 .
$$

Hence if $m_{i}=1$ then $\mu_{i 1}(E)=\alpha^{(p-1) / 2}(E)= \pm 1$. Since $\&$ is not of type $L_{2}(p)$, $E \neq 1$. Thus for any $k$ either $\alpha^{k}(E) \neq \alpha^{(p-1) / 2}(E)$ or $\alpha^{k+1}(E) \neq \alpha^{(p-1) / 2}(E)$. Consequently (4.5) and (4.6) imply that at most $\frac{p+1-2 s}{2}$ of $m_{i}^{\prime}$ s are equal to 1.

Suppose first that $2 s-1<p-s=d$. The minimality of $d$ and (4.6) yield that $m_{i} \neq 0$ for $i=1, \ldots, s-1$. Thus by $(4.6)$

$$
s-1 \leq \frac{p+1-2 s}{2}+\frac{1}{2}\left\{p-2 s-\frac{(p+1-2 s)}{2}\right\}=\frac{1}{4}(3 p-6 s+1) .
$$

Hence $s \leq \frac{3 p+5}{10}$ and so $d=p-s \geq \frac{7 p}{10}-\frac{1}{2}$ as required.

Assume now that $2 s-1 \geq p-s$. Thus $s \geq 5$. The minimality of $d$ yields that $m_{i} \neq 0$ for $i=1, \ldots, s-2$.

Thus by (4.6)

$$
s-2 \leq \frac{p+1-2 s}{2}+\frac{1}{2}\{p-2 s-(p+1-2 s)\}=\frac{1}{4}(3 p-6 s+1)
$$

Therefore

$$
10 s \leq 3 p+9=9 s+6
$$

Hence $s \leq 6$ and $p \neq 13$ so $p \leq 3 s-1 \leq 17$. Thus $s=6$ and $p=17$. Furthermore $\operatorname{dim}_{K} L_{5}=11=d$. Since $\mathscr{S}$ is in the kernel of $L_{5}$ it may be assumed $L$ was chosen initially such that $\mathfrak{S}$ is in the kernel of $L$. Hence since $L$ is faithful it may be assumed that $\tilde{D}=\langle 1\rangle$. Thus $L$ is in the principal $p$-block. The minimality of $d$ implies that $L$ is an irreducible $K(S$-module. Therefore $|\langle E\rangle|=|\mathfrak{N}: \mathfrak{P}|>2$ by (2.3). Thus for any $k$ either $\alpha^{k}(E) \neq \alpha^{(p-1) / 2}(E)$ or $\alpha^{k+1}(E) \neq \alpha^{(p-1) / 2}(E)$ or $\alpha^{k+2}(E) \neq \alpha^{(p-1) / 2}(E)$. Thus by (4.9) at most $\frac{p+2-2 s}{3}<3$ of the $m_{i}$ 's are equal to 1 and so by (4.6).

$$
4=s-2 \leq 2+\frac{1}{2}(5-2)<4
$$

This contradịction establishes Theorem 1 in all çases. 


\section{$\S 5$. Proof of Theorem 2}

Throughout this section $\mathbb{B}$ is a group which satisfies the hypotheses but not the conclusion of Theorem $2 . \mathfrak{F}$ is a $S_{p}$-subgroup of $\mathbb{B}$ and $\Re=\mathrm{N}_{\mathfrak{S}}(\mathfrak{B}) . \quad \zeta$ is an irreducible faithful complex character of degree $d$.

LEMMA 5.1. $\mathbb{B}$ is simple. $|\mathfrak{P}|=p$ and $\mathbf{C}_{\mathfrak{S}}(\mathfrak{B})=\mathfrak{P}$

Proof. Let $\mathbb{B}_{0}$ be the subgroup of $\mathbb{B}$ generated by all $p$-elements in $\mathbb{B}$. Thus $\mathbb{B}_{0} \triangleleft \mathbb{B}$. Let $\zeta_{\mid \mathscr{S}_{0}}=\sum_{i=1}^{n} \omega_{i}$ where each $\omega_{i}$ is an irreducible character of $\mathbb{B}_{0}$. Since the $\omega_{i}$ are conjugate under the action of $\&$ they all have the same degree. Thus if $n>1, \omega_{i}(1)<\frac{p-1}{2}$ for each $i$ and so by [5] $\gg \triangleleft \&$ contrary to assumption. Hence $\zeta \mid \Theta_{0}=\omega$ is irreducible. Thus $\mathbf{Z}\left(\mathbb{S}_{0}\right)=\mathbf{Z}(\mathbb{B})=\langle 1\rangle$.

Suppose that $|\mathfrak{P}| \neq p$. Then there exists $\mathfrak{P}_{1} \triangleleft \mathbb{S}$ with $\left|\mathfrak{P}: \mathfrak{P}_{1}\right|=p \quad[4]$. Hence $\mathfrak{P} \subseteq \mathbf{C}_{\mathscr{S}}\left(\mathfrak{B}_{1}\right) \triangleleft \mathbb{B}$ and so $\mathfrak{B}_{0} \subseteq \mathbf{C}_{\mathscr{S}}\left(\mathfrak{B}_{1}\right)$. Thus $\mathfrak{\beta}_{1} \subseteq \mathbf{Z}\left(\mathfrak{B}_{0}\right)=\langle 1\rangle$ and so $|\mathfrak{P}|=p$.

Suppose that $\mathfrak{H} \triangleleft \mathbb{S}_{0}, \mathfrak{H} \neq \mathbb{S}_{0}$ Then $\mathfrak{U}$ is a $p^{\prime}$-group. Hence $\mathfrak{H} \triangleleft \mathfrak{H P}$ and $\mathfrak{H} \mathfrak{P}$ is $p$-solvable. Since $\mathfrak{Y P}$ has a faithful complex representation of degree $d<p-1$ it follows that $\mathfrak{F P}$ has a $K$-representation whose kernel is in $\mathfrak{F}$ for a suitable field $K$ of characteristic $p$. Thus by Theorem $B$ of Hall and Higman [7] (see also [11] for a simplification of part of the proof.) $\mathfrak{B} \subseteq \mathbf{C}_{\mathscr{S}}(\mathfrak{U}) \triangleleft \mathfrak{S}_{0}$. Thus $\mathfrak{A} \subseteq \mathbf{Z}\left(\mathbb{B}_{0}\right)=\langle 1\rangle$. Therefore $\mathbb{B}_{0}$ is simple.

By (2.2)

$$
e=|\mathfrak{R}: \mathbf{C}(\mathfrak{B})|=p-\zeta(1)=p-\omega(1)=\left|\mathbf{N}_{\mathfrak{S}_{0}}(\mathfrak{R}): \mathbf{C}_{\mathfrak{S}_{0}}(\mathfrak{R})\right| .
$$

Since $\mathbb{B}=\mathbb{B}_{0} \mathfrak{N}$ this yields that $\mathbb{B}=\mathbb{B}_{0} \mathrm{C}_{\mathscr{S}}(\mathfrak{P})$. If $\mathbb{B}$ is not of type $L_{2}(p)$ then Theorem 1 implies that $\mathbb{B}=\mathbb{B}_{0}$ and $\mathfrak{P}=\mathbf{C}_{\mathscr{S}}(\mathfrak{B})$ completing the proof of the Lemma. Suppose that $\&$ is of type $L_{2}(p)$. Thus $\mathbb{B}_{0} \approx P S L_{2}(p)$. Since $P S L_{2}(p)$ admits no outer automorphism which leaves all the elements in a $S_{p}$-subgroup fixed it follows that $\mathbb{B}=\mathfrak{B}_{0} \approx P S L_{2}(p)$. Thus $\mathbb{B}$ is simple since $p>3$ and $\mathbf{C}_{\mathscr{S}}(\mathfrak{B})=\mathfrak{F}$ as required.

Let $F$ be a finite extension field of the field of $p$-adic numbers which is a splitting field for $B$ and all its subgroups and contains all the $|\dot{B}|$ th roots of unity. Let $R$ be the ring of local integers in $F$, let $p$ be the maximal ideal in $R$ and let $K=R / p$. It is well known that there exists an $R \&$-module $Z$ which affords the character $\zeta$. Let $\bar{Z}=Z / \mathrm{p} Z$.

Lемма 5,2. $\bar{Z}$ is absolutely irreducible. 
Proof. Since $F$ contains all $|\mathbb{S}|$ th roots of unity $K$ is a splitting field of B. Thus it suffices to show that $\bar{Z}$ is irreducible. By (2.2) and Lemma 5.1 every modular irreducible constituent of $\bar{Z}$ is faithful. Hence if $\bar{Z}$ is reducible then $\&$ has a faithful $K$-representation of degree at most $d / 2<\frac{p-1}{2}$. Hence by Theorem $1 \&$ is of type $L_{2}(p)$ and so $\&$ is isomorphic to $P S L_{2}(p)$ by Lemma 5.1. In this case it is well known that $e=\frac{p-1}{2}$ and $p \equiv 1(\bmod 4)$ contrary to assumption.

Let $\mathfrak{R}=\mathfrak{P} \mathfrak{F}$ with $\mathfrak{B} \cap \mathbb{E}=\langle 1\rangle$ and let $(\xi=\langle E\rangle$. Let $\alpha$ be defined as in (3.1). Let $\varepsilon$ be a primitive $e^{t h}$ root of unity in $R$ such that the image of $\varepsilon$ in $R / p$ is $\alpha(E)$.

LeмmA 5.3. $\bar{Z}_{\mid \Re \neq} \neq V_{p-e}^{1}$

Proof. Suppose that $\bar{Z}_{\mid \mathfrak{R}} \approx V_{p-e}^{1}$. Let $\left\{\zeta_{i} \mid i=1, \ldots, \frac{p-1}{e}\right\}$ be all the irreducible complex characters of $\$$ which are algebraically conjugate to $\zeta$. Then by $(2.2)$ the $\zeta_{i}$ are all equal as Brauer characters. Thus if $U$ is an $R \mathbb{S}$ module affording the character $\emptyset$ such that $\bar{U}=U / p U$ is the projective indecomposable $K \otimes$-module corresponding to $\bar{Z}$ then $\Phi=\sum_{i=1}^{p-1 / e} \zeta_{i}+\theta$ for some character $\theta$. Thus [11, Theorem 1] there exists an $R \$$-module $M$ which affords the character $\sum_{i=1}^{p-1 / e} \zeta_{i}$ such that $\bar{M}=M / p M$ is indecomposable. Since $\operatorname{dim}_{K} \bar{M}=$ $\left(\frac{p-1}{e}-1\right) p+1$ Higman's theorem and Lemma 3.1 imply that

$$
\bar{M}_{\mid \mathfrak{R}} \approx V_{1}^{\alpha^{k}}+\sum_{\jmath=1}^{(p-1) / \ell-1} V_{p}^{\alpha}{ }^{\alpha(\jmath)}
$$

for suitable $k$ and $a(j)$. Let $\psi$ be the Brauer character afforded by $\bar{M}$. Then Lemma 3.3 implies that

$$
\begin{aligned}
& \psi(E)=\varepsilon^{k}+\sum_{j=1}^{(p-1) / e-1} \varepsilon^{a(j)}\left(\sum_{t=0}^{p-1} \varepsilon^{-t}\right)=\varepsilon^{k}+\sum_{j=1}^{(p-1) / e-1} \varepsilon^{a(j)} \\
& \zeta_{i}(E)=\sum_{t=0}^{p-e-1} \varepsilon^{-t}=1
\end{aligned}
$$

Since $\psi(E)=\sum_{i=1}^{(p-1) / e} \zeta_{i}(E)$ this yields that $k=1$ and $a(j)=1$ for all $j$. Hence $\bar{M} \mid \mathfrak{N}$ $\approx V_{1}^{1}+A$ for some projective $K \Re$-module $A$. Let $L_{0}$ be the trivial 1-dimensional $K\left(3\right.$-module. Then $\left.L_{0}\right|_{\mathfrak{R}} \approx V_{1}^{1}+B$ for some projective $K \Re_{i}$-module $B$. Hence by Higman's Theorem $\bar{M}$ and $L_{0}$ are both direct summands of $\left(V_{1}^{1}\right)^{\text {(S) }}$ contrary to the Mackey decomposition. This contradiction establishes the lemma. 
Lemma 5.4. $e \equiv 0(\bmod 2), \bar{Z}_{\mid \Re} \approx V_{p-1}^{\alpha^{e / 2}}$ and $\frac{p-1}{e} \equiv 0(\bmod 2)$

Proof. Let $\bar{Z}_{\mid \mathfrak{R}} \approx V_{p-e}^{\alpha^{k}}$. By Lemma $3.3 \zeta(E)=\varepsilon^{k}$. Since $\mathbf{C}_{\mathfrak{G}}(\mathfrak{P})=\mathfrak{P}(2.2)$ implies that $\zeta(E)$ is rational. Hence $\varepsilon^{k}= \pm 1$. If $\varepsilon^{k}=1$ then $e \mid k$ and so $\bar{Z}_{\mid \Re} \approx V_{p-e}^{1}$ contrary to Lemma 5.3. Hence $\varepsilon^{k}=-1$. Therefore $e \equiv 0(\bmod 2)$ and $\bar{Z}_{\mid \Re} \approx V_{p-e}^{\alpha^{e / 2}}$.

Since $\mathbb{B}$ is simple $\operatorname{det}_{p-s}^{\alpha^{e / 2}}(E)=1$. Thus by Lemma 3.3

$$
1=\alpha^{e / 2(p-e)} \alpha^{-(p-e)(p-e-1) / 2}(E)=-\alpha^{-(p-e)(p-e-1) / 2}(E)=-\alpha^{-(p-e-1) / 2}(E) .
$$

Thus $\frac{p-e-1}{2} \equiv e / 2(\bmod e)$ and so $\frac{p-1}{2} \equiv 0(\bmod e)$. Hence $\frac{p-1}{e} \equiv 0$ $(\bmod 2)$ as required.

Theorem 2 now follows from Lemmas 5.1 and 5.4.

\section{REFERENCES}

[1] Brauer, R., Investigations on group characters, Ann. of Math. 42 (1941), 936-958.

[2] Brauer, R., On groups whose order contains a prime number to the first power I, II, Am. J. Math. 64 (1942), 401-420, 421-440.

[ 3 ] Curtis and Reiner, Representation Theory of Finite Groups and Associative Algebras, Interscience 1962 .

[4] Feit, W., Groups which have a faithful representation of degree less than $p-1$, Trans. of A.M.S. 112 (1964), 287-303.

[ 5 ] Feit, W. and Thompson, J. G., Groups which have a faithful representation of degree less than $\frac{p-1}{2}$. Pacific J. Math. 11 (1961), 1257-1262.

[6] Green, J. A., The modular representation algebra of a finite group, Ill. J. Math. 6 (1962), 607-619.

[ 7 ] Hall, P. and Higman, G., On the $p$-length of $p$-soluble groups and reduction theorems for Burnside's problem, Proc. London Math. Soc. 6 (1956), 1-42.

[ 8 ] Janko, Z., A new finite simple group with abelian 2-Sylow subgroups (to appear).

[9] Schur, I., Über die darstellung der symmetrischen und der alternierenden gruppen durch gebrochene lineare Substitutionen, J. Für Math. 139 (1911), 155-250.

- [10] Swan, R. G., Periodic resolutions for finite groups, Ann. of Math. 72 (1960), 267-291.

[11] Thompson, J. G., Vertices and Sources (to appear).

[12] Tuan, H. F., On groups whose orders contain a prime to the first power. Ann. of Math. 45 (1944), 110-140.

Yale University 